\title{
Multifaceted (Mecsus) Protocol for Microbial Metabolites Studies
}

\author{
Rasha Saad Abdelgadir Soliman, ${ }^{1, *}$, Sadia Sultan ${ }^{1}$, Kalavathy Ramasamy ${ }^{2}$, Syed Adnan Ali Shah ${ }^{1}$, \\ Cole A.L.J ${ }^{3}$ and J.-F.F. Weber ${ }^{1, *}$ \\ ${ }^{1}$ Atta-ur-ahman Research Institute for the Study of Natural Drugs (RiND), Faculty of Pharmacy, Universiti Teknologi \\ MARA, 42300 Puncal Alam, Selangor, Malaysia; ${ }^{2}$ Faculty of Pharmacy, Universiti Teknologi MARA, 42300 Puncal \\ Alam, Selangor, Malaysia; ${ }^{3}$ School of biological sciences, University of Canterbury, Christchurch, New Zeland; \\ E-mails:rshoo70@yahoo.comorjffweber@puncakalam.uitm.edu.my
}

The advent of new chemical genetic tools and high-throughput screening technologies and platforms have already led and will continue to lead in accessing the natural product diversity of microorganisms. This paper represents a continuation of the work on the investigation into means of making better use of the advantages of new technologies and approaches in drug discovery that the Atta-ur-Rahman Research Institute of Natural Product Discovery (RiND) at MARA University of Technology has engaged in. MECSUS (Microtiter plate, Elicitors, Combination, Solid phase extraction, UHPLC, Statistical analysis) is a protocol for microbial metabolites studies that involves miniaturized parallel fermentations in 96-well microtiter plate with up to ninety six different media, parallel extraction and data analysis. The aforementioned protocol introduces elements of incremental novelty in the natural product screening program by means of combining and harnessing existing ideas, techniques, and technologies into a protocol for the implementation of the OSMAC (one strain many compounds) approach at micro-scale. Its main advantage is the decrease of the scale of operation with the use of the 96-well microtiter plates. It's benefits include the possibility of overcoming few issues such as processing time and human resources that have somewhat hampered the implementation of the OSMAC approach and/or the systematic study of a large library of microorganisms.

Keywords: MECSUS, microbial metabolites, and micro-scale. 